\title{
BANCO PÚBLICO DE AGUA PARA RECUPERAR AGUA PARA EL MEDIOAMBIENTE EN SITUACIÓN DE SEQUÍA EN CONTEXTO DE MONOPOLIO-MONOPSONIO
}

\author{
Montilla-López, N.M. (1), Gómez-Limón, J.A. (2) (P), Gutiérrez-Martín, C. (3)
}

\begin{abstract}
${ }^{1}$ Profesora Sustituta Interina, Universidad de Córdoba, Grupo de Investigación WEARE: Water, Environmental and Agricultural Resources Economics, g02molon@uco.es

${ }^{2}$ Catedrático de Universidad, Universidad de Córdoba, Grupo de Investigación WEARE: Water, Environmental and Agricultural Resources Economics, jglimon@uco.es

${ }^{3}$ Profesor Titular de Universidad, Universidad de Córdoba, Grupo de Investigación WEARE: Water, Environmental and Agricultural Resources Economics, carlos.gutierrez@uco.es
\end{abstract}

\section{Resumen}

Los mercados de derechos temporales de agua (mercados de dotaciones) son instrumentos ampliamente utilizados para recuperar agua para el medioambiente durante los períodos de escasez coyuntural (sequías). Este documento propone un banco de agua que opera en un contexto de monopolio-monopsonio con el doble propósito de reasignar el agua entre los agricultores y adquirir agua para el medioambiente durante los períodos de sequía. El banco de agua propuesto sería una mejora en los procedimientos actuales para la compra pública de dotaciones de agua con fines ambientales. Este sería administrado por una agencia pública que buscaría maximizar la eficiencia económica generada en las compras y ventas de agua por los regantes y la eficiencia generada por la recuperación de las dotaciones de agua para el medioambiente. Una característica adicional e innovadora del análisis realizado es que considera las ineficiencias en la economía en su conjunto causadas por el gasto público en compras de dotaciones de agua, medido a través del coste marginal de los fondos públicos. El rendimiento potencial del banco de agua propuesto se simula mediante técnicas de programación matemática, tomando la cuenca del río Guadalquivir (sur de España) como caso de estudio. Los resultados evidencian que, en términos de eficiencia económica, el banco propuesto supera a los instrumentos actualmente en vigencia para comprar dotaciones de agua.

\section{Introducción y objetivos}

En cuencas cerradas, donde no es posible aumentar más la oferta de agua (Molle et al. 2010), el medioambiente a menudo se ve afectado negativamente debido a que las extracciones superan los límites sostenibles. Este problema es especialmente agudo durante los períodos de sequía, cuando todos los derechos de agua están asignados y no es posible atender a todos los usos, incluidos los ambientales (Wheeler et al. 2013). En estas situaciones de escasez coyuntural, la implementación de bancos de agua puede resultar una alternativa de gestión útil (Hanak \& Stryjewski 2012). Estos bancos, conforme a la legislación española son instituciones de naturaleza pública, donde los organismos de cuenca se encargan de centralizar todas las operaciones del mercado, tanto de compras como de ventas de dotaciones. A través de estas operaciones, las autoridades pueden comprar dotaciones de agua con fondos públicos para mejorar los caudales de los ríos afectados por la sequía.

El objetivo propuesto en este trabajo es doble: i) proponer un banco de agua en régimen de monopolio-monopsonio que facilite reasignar agua entre usos productivos y adquirir 
dotaciones para mejorar la calidad ambiental de las masas de agua con el propósito de maximizar la eficiencia económica total, y ii) analizar el desempeño potencial del banco de agua propuesto en la cuenca del Guadalquivir cuantificando las mejoras de eficiencia económica que se alcanzaría con su implementación.

\section{Materiales y métodos}

A diferencia de los bancos de agua que operan para recuperar agua ambiental comprando agua en un mercado competitivo, el banco de agua público propuesto actuaría en régimen de monopolio-monopsonio, prohibiendo por tanto todos los contratos de cesión, de manera que todas las operaciones de mercado se centralizasen a través del banco. Este poder de mercado por parte del banco permitiría a la agencia gestora del mismo distinguir entre los usos del agua (productivos y ambientales), lo que posibilitaría que el valor marginal del agua vendida a los usuarios productivos $\left(P_{v}\right)$ difiriese del valor marginal del agua destinada a los usos ambientales $\left(V_{e}\right)$. Como institución pública sin ánimo de lucro, se supone que la agencia del agua usaría este poder de mercado para maximizar la eficiencia económica, cubriendo cualquier déficit en el flujo de caja del banco (ingresos obtenidos por las ofertas públicas de venta menos gastos en ofertas públicas de compra) con fondos públicos.

Operando en régimen de monopolio-monopsonio, el banco de agua podría crear un diferencial entre el precio a pagar por las adquisiciones y el precio a percibir por las ventas de dotaciones, lo que le permitirá autofinanciar parte de las adquisiciones de agua para fines ambientales, tal y como se muestra en la Figura 1. En esta figura se observa: a) la oferta de agua del banco $\left(Q_{c}\left(P_{c}\right)\right)$, que indica las cantidades que el banco puede comprar a los regantes de la cuenca para cada precio $P_{c}$; b) la demanda del sector agrario $\left(Q_{v}\left(P_{v}\right)\right)$, que cuantifica el volumen de las dotaciones que los regantes de la cuenca están dispuestos a comprar para cada precio $P_{v}$; c) la demanda ambiental de agua $\left(Q_{e}\left(V_{e}\right)\right)$, que establece la cantidad de agua que la sociedad está dispuesta a adquirir con fines ambientales para cada valor $V_{e}$; y d) la demanda agregada agraria y ambiental $\left(Q_{t}\right)$.

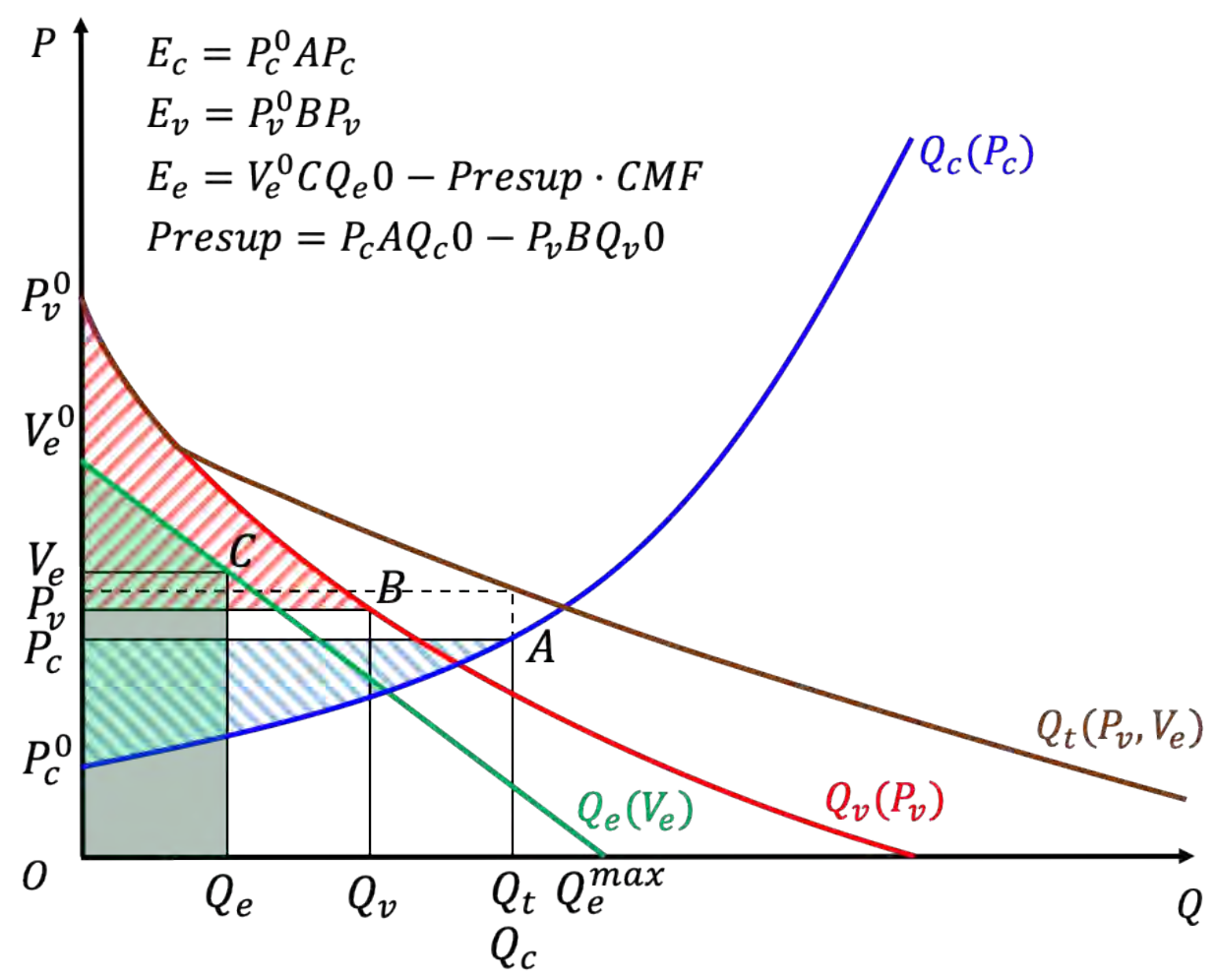

Figura 1. Funcionamiento del banco de agua. 
La estrategia del banco de agua para autofinanciar parcialmente las compras de agua para el medioambiente consiste en comprar una cantidad de agua $Q_{c}$ a un precio $P_{c}$, para posteriormente revender parte de dicha cantidad, $Q_{v}$, a un precio $P_{v}$ mayor. La diferencia entre el agua adquirida y vendida, $Q_{e}$, es destinada para fines ambientales. Los precios (y las cantidades) de compra y venta se establecen por la agencia pública que gestiona el banco que, como se ha indicado, busca maximizar el bienestar social, cuantificado como la eficiencia económica total $(E)$. Esta eficiencia se ha calculado como suma de los excedentes económicos generados por las compras de agua, las ventas y la recuperación de agua con fines medioambientales.

En este sentido, la eficiencia generada por la oferta pública de compra se puede calcular a través del excedente del productor $\left(E_{c}\right)$, que mide el beneficio obtenido por los usuarios del agua cuando venden sus dotaciones de agua al banco (la diferencia entre los ingresos por ventas y los ingresos no percibidos debido a un menor uso de agua). Esta eficiencia está representada por el área rallada en rojo $P_{c} A P_{c}^{0}$. La eficiencia generada por las ventas del banco a usuarios productivos se calcula a través del excedente del consumidor $E_{v}$, lo que representa el beneficio que obtienen los usuarios productivos cuando compran agua al banco (la diferencia entre el ingreso adicional debido a una mayor producción y el coste de compra). Si el precio de venta se fija en $P_{v}$, entonces $E_{v}$ es igual al área $P_{v}^{0} B P_{v}$.

La recuperación de agua para fines ambientales también genera ganancias de eficiencia, que pueden explicarse utilizando el concepto de excedente del consumidor como en el caso de los usuarios productivos. En este caso, el excedente ambiental $E_{e}$ mide el beneficio que la sociedad obtiene del agua recuperada para el medioambiente como la diferencia entre la eficiencia económica adicional debido a los mayores flujos en las masas de agua y el presupuesto necesario para recuperar esa agua. Si el valor del agua ambiental para la sociedad se fija en $V_{e}$, entonces $E_{e}$ es igual al área $V_{e}^{0} C Q_{e} O$. Sin embargo, debe tenerse en cuenta que los recursos presupuestarios necesarios para implementar cualquier política de gasto público primero deben recaudarse a través del sistema tributario, y esto inevitablemente causa distorsiones que reducen la eficiencia económica. Estas pérdidas pueden cuantificarse a través del coste marginal de los fondos públicos $(C M F)$, un parámetro que refleja el precio sombra que la sociedad paga por cada unidad monetaria invertida en políticas de gasto público (Dahlby 2008). Por lo tanto, el excedente ambiental $E_{e}$ se puede medir con mayor precisión como la diferencia entre la eficiencia generada por la cantidad de agua destinada para fines ambientales (área por debajo de la curva de demanda de la sociedad para el agua ambiental, $\left.V_{e}^{0} C Q_{e} O\right)$ y el coste social de esta agua en términos presupuestarios (Presupuesto $\times$ CMF).

El análisis empírico realizado se basa en las curvas de oferta agregadas de agua de los usuarios agrarios $Q_{c}\left(P_{c}\right)$ y las curvas de demanda agregada de los regantes $Q_{v}\left(P_{v}\right)$ en la cuenca del río Guadalquivir estimadas por Gutiérrez-Martín et al. (2020). Estos autores basan sus estimaciones en los resultados de los modelos de programación matemática construidos por Montilla-López et al. (2018) para simular el comportamiento de los regantes frente a un banco de agua (decisiones sobre la venta y compra de dotaciones de agua) en esta misma cuenca. Gutiérrez-Martín et al. (2020) estiman curvas de oferta y demanda agregadas para tres escenarios diferentes de disponibilidad de agua (intensidad de las sequías): sequía "moderada", "severa" e "intensa", donde las dotaciones de agua asignadas a los regantes son el $75 \%, 50 \%$ y $25 \%$ de las otorgadas en un año hidrológico promedio, respectivamente.

La cuenca del Guadalquivir se considera una cuenca cerrada a nuevos usos debido a que no es posible aumentar la oferta de agua. En este sentido, la reforma de la Ley de Aguas de 1999 introduce instrumentos de mercado para la gestión de la demanda, entre los que se encuentran los mercados de agua y los centros de intercambio de derechos de agua (bancos de agua). El uso medio de agua en la cuenca es de $3.815 \mathrm{hm}^{3} / a n ̃ o$, de los que el $88 \%$ se destina al regadío. Su clima mediterráneo expone la cuenca a periodos cíclicos de sequías, 
durante los cuales las dotaciones de todos los usuarios agrarios son reducidas proporcionalmente, ya que los usos urbanos están asegurados por el sistema de prioridad establecido. Por tanto, son los usuarios agrarios los únicos interesados en participar en el mercado establecido a través del banco de agua.

Siguiendo a Gutiérrez-Martín et al. (2020), la demanda social por el agua ambiental $Q_{e}\left(V_{e}\right)$ se asume que es lineal, conectando el punto de agua ambiental nula, para el cual el valor marginal del agua ambiental es máximo $\left(Q_{e}=0, V_{e}^{0}\right)$, y el punto de máxima agua ambiental demanda, para el que lógicamente el valor marginal del agua ambiental es cero $\left(Q_{e}^{\max }, V_{e}=0\right)$ (véase ecuación 8). Se ha considerado que la máxima agua ambiental demanda $\left(Q_{e}^{\max }\right)$ se corresponde con la diferencia entre el flujo de agua en un año hidrológico normal y el flujo de mínima agua ambiental correspondiente a los caudales ecológicos. Esta diferencia para la cuenca del Guadalquivir se ha estimado en $6.787 \mathrm{hm}^{3} /$ año. Sin embargo, no hay estudios que analicen el valor marginal máximo del agua ambiental $\left(V_{e}^{0}\right)$ que la sociedad está dispuesta a pagar como máximo por el agua ambiental para las distintas situaciones de disponibilidad de agua. Por ese motivo, se ha optado por parametrizar el valor de $V_{e}^{0}$ al objeto de determinar el comportamiento del banco de agua en función del valor de este parámetro.

Para simular el rendimiento potencial de esta propuesta institucional de banco de agua, se ha construido un modelo de programación matemática cuyo objetivo es maximizar la eficiencia total, y cuyas variables de decisión son los precios de adquisición y venta de las dotaciones de agua, que a su vez determinarán las cantidades compradas y vendidas, así como recuperadas para el medioambiente. El modelo de simulación así construido es el que sigue:

$\operatorname{Max} E=E_{c}+E_{v}+E_{e}$

Sujeto a:

$$
\begin{aligned}
& E_{c}=\int_{P_{c}^{0}}^{P_{c}} Q_{c}\left(P_{c}\right) d P_{c} \\
& E_{v}=\int_{P_{v}}^{P_{v}^{0}} Q_{v}\left(P_{v}\right) d P_{v} \\
& E_{e}=\int_{V_{e}}^{V_{e}^{0}} Q_{e}\left(V_{e}\right) d V_{e}+Q_{e} V_{e}-\text { Presup } \cdot C M F \\
& Q_{c}=Q_{v}+Q_{e} \\
& Q_{c}=a_{c} P_{c}^{2}+b_{c} P_{c}+c_{c} \\
& Q_{v}=a_{v} P_{s}^{2}+b_{v} P_{v}+c_{v} \\
& Q_{e}=Q_{e}^{\max }\left(1-\frac{V_{e}}{V_{e}^{0}}\right) \\
& P_{c} \cdot Q_{c}=P_{v} \cdot Q_{v a}+\text { Presup }
\end{aligned}
$$

Las eficiencias de las compras, las ventas y ambiental está representado por las ecuaciones 2-4. La ecuación 5 muestra el equilibrio cuantitativo de agua. Las ecuaciones 6,7 y 8 representan las curvas de oferta $\left(Q_{c}\right)$ y demanda agraria $\left(Q_{v}\right)$ y ambiental $\left(Q_{e}\right)$, respectivamente. Finalmente, la ecuación 9 muestra el equilibrio presupuestario, igualando gastos e ingresos del banco de agua. 


\section{Resultados y discusión}

La Figura 2 muestra los resultados obtenidos para el banco de agua para los tres escenarios de escasez de agua propuestos en términos de volúmenes negociados, precios de compraventa, fondos públicos requeridos y mejoras de eficiencia con relación a un escenario de referencia sin comercio para cada valor marginal máximo de agua ambiental $\left(V_{e}^{0}\right)$ que varía de 0 a $0,8 € / \mathrm{m}^{3}$. Los tres escenarios considerados revelan ganancias de eficiencia netas en comparación con el escenario de referencia, aunque con algunas diferencias.
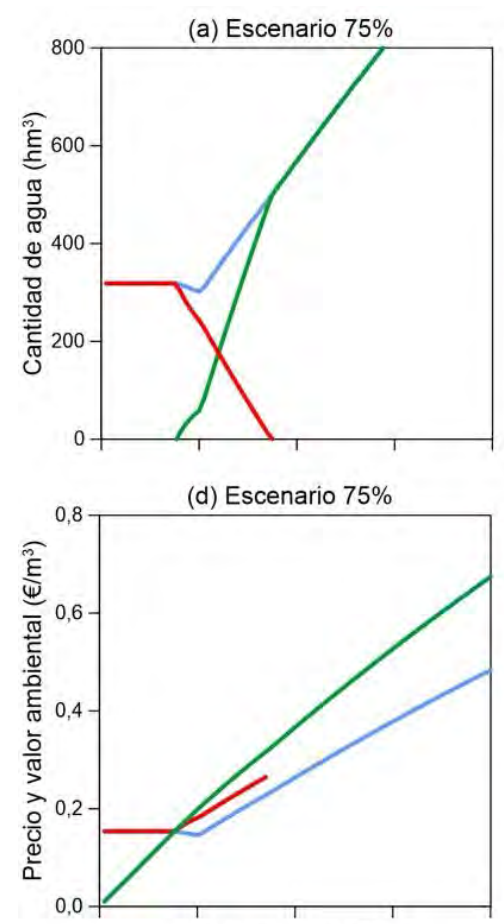

(g) Escenario $75 \%$
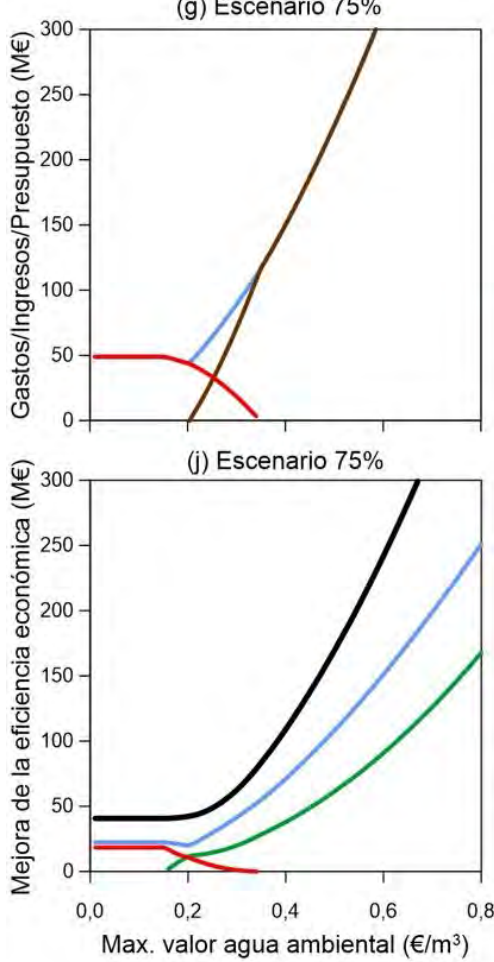

(b) Escenario $50 \%$

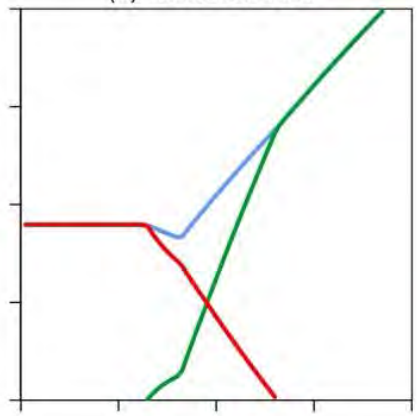

(e) Escenario $50 \%$

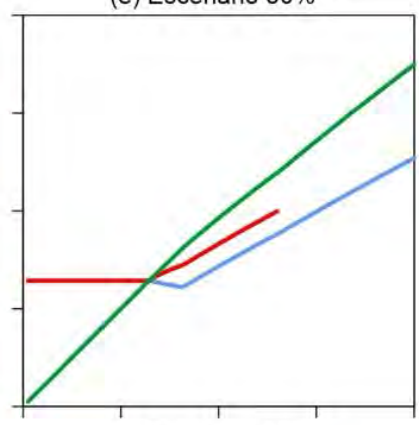

(h) Escenario 50\%

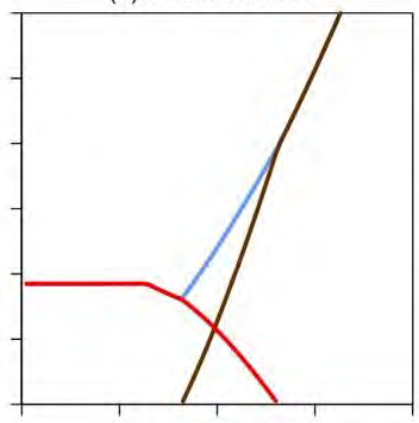

(k) Escenario $50 \%$

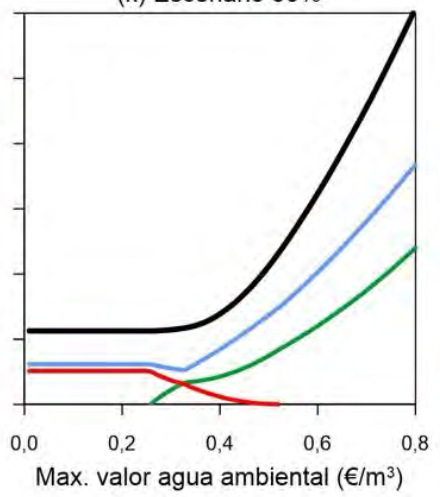

(c) Escenario $25 \%$

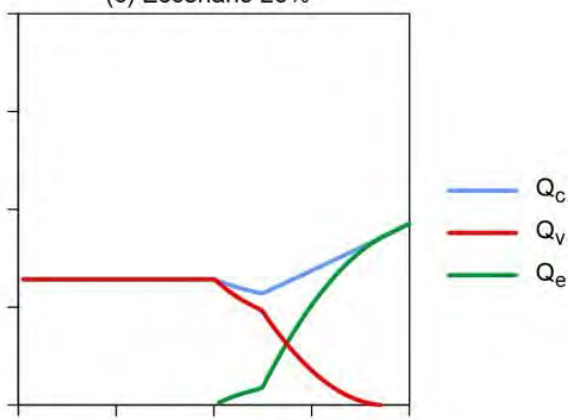

(f) Escenario 25\%

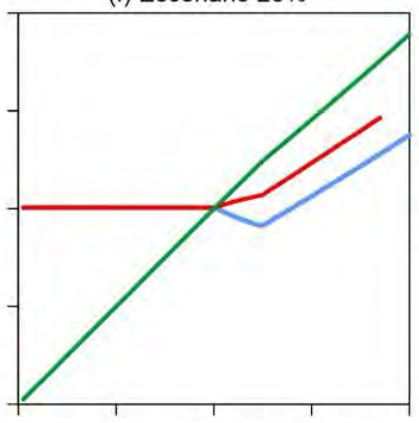

(i) Escenario 25\%

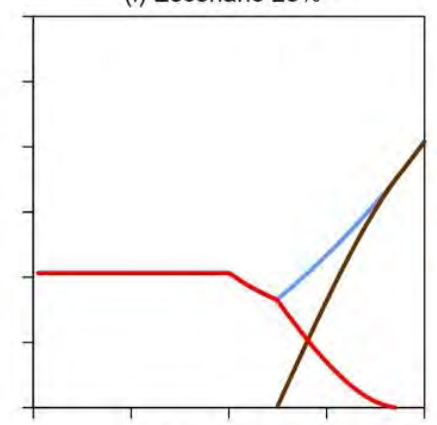

(I) Escenario 25\%

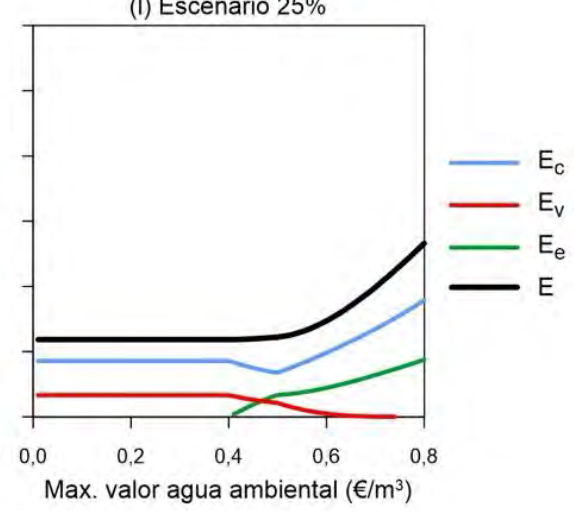

Figura 2. Resultados para el banco de agua: volúmenes negociados, precios, flujos de efectivo y mejoras de eficiencia. 
La parametrización de $V_{e}^{0}$ muestra cuatro fases definidas en todos los escenarios considerados. Las principales características de cada fase se resumen en la Tabla 1. La primera fase es la correspondiente a los valores más bajos de $V_{e}^{0}$, y se caracteriza por el hecho de que no se recupera agua para el medioambiente (ver Figura 1a-c), ya que el valor marginal del agua ambiental está por debajo del precio de equilibrio del mercado $\left(P_{c}=P_{v}\right.$, ver Figura $1 \mathrm{~d}$-f). De esta forma, el banco de agua compra dotaciones de agricultores que están dispuestos a vender y posteriormente las vende todas $\left(Q_{c}=Q_{v}\right)$ a otros agricultores al mismo precio. Puesto que los gastos por las compras igualan a los ingresos obtenidos por las ventas, no se requieren fondos públicos para las operaciones (ver Figura 1g-i).

Tabla 1. Fases del banco de agua en función del máximo valor social del agua ambiental $\left(V_{e}^{0}\right)$

\begin{tabular}{|c|c|c|c|c|c|}
\hline Fase & $\begin{array}{l}\text { Rang } \\
\text { A75\% }\end{array}$ & $\begin{array}{r}\text { o de } V_{e}^{\mathbf{0}} \\
A 50 \%\end{array}$ & $\begin{array}{l}\left.A / \mathrm{m}^{3}\right) \\
A 25 \%\end{array}$ & Descripción operaciones & $\begin{array}{l}\text { Relaciones } \\
\text { matemáticas }\end{array}$ \\
\hline Fase 1 & $\begin{array}{l}0,00- \\
0,15\end{array}$ & $\begin{array}{l}0,00- \\
0,25\end{array}$ & $\begin{array}{l}0,00- \\
0,40\end{array}$ & $\begin{array}{l}\text { El banco compra a regantes y revende toda el } \\
\text { agua a otros regantes. No se recupera nada para } \\
\text { el medioambiente. El banco se autofinancia. }\end{array}$ & $\begin{array}{l}V_{e}<P_{c}=P_{v} \\
Q_{c}=Q_{v} \\
Q_{e}=0 \\
\text { Presup }=0\end{array}$ \\
\hline Fase 2 & $\begin{array}{l}0,16- \\
0,20\end{array}$ & $\begin{array}{l}0,26- \\
0,32\end{array}$ & $\begin{array}{c}0,41- \\
0,49\end{array}$ & $\begin{array}{l}\text { El banco compra a regantes; parte la revende a } \\
\text { otros regantes y parte se destina al medioam- } \\
\text { biente. El banco se autofinancia empleando su po- } \\
\text { der de monopolio-monopsonio. }\end{array}$ & $\begin{array}{l}P_{c}<P_{v}<V_{e} \\
Q_{c}=Q_{v}+Q_{e} \\
Q_{e}>0 \\
\text { Presup }=0\end{array}$ \\
\hline Fase 3 & $\begin{array}{l}0,21- \\
0,34\end{array}$ & $\begin{array}{l}0,33- \\
0,52\end{array}$ & $\begin{array}{l}0,50- \\
0,74\end{array}$ & $\begin{array}{l}\text { El banco compra a regantes; parte la revende a } \\
\text { otros regantes y parte se destina al medioam- } \\
\text { biente. El banco emplea su poder de mercado, } \\
\text { pero necesita asignación de presupuesto público. }\end{array}$ & $\begin{array}{l}P_{c}<P_{v}<V_{e} \\
Q_{c}=Q_{v}+Q_{e} \\
Q_{e}>0 \\
\text { Presup }>0\end{array}$ \\
\hline Fase 4 & $>0,35$ & $>0,53$ & $>0,75$ & $\begin{array}{l}\text { El banco compra a regantes y destina toda el agua } \\
\text { al medioambiente. El banco emplea su poder de } \\
\text { monopolio-monopsonio, pero necesita asignación } \\
\text { de presupuesto público. }\end{array}$ & $\begin{array}{l}P_{c}<V_{e} \\
Q_{c}=Q_{e} \\
Q_{v}=0 \\
\text { Presup }>0\end{array}$ \\
\hline
\end{tabular}

Después de que el valor máximo del agua ambiental $\left(V_{e}^{0}\right)$ alcance los precios de equilibrio del mercado correspondientes en cada escenario, los resultados de la simulación entran en una segunda fase, donde una parte de las asignaciones de agua compradas a los agricultores se recupera para el medioambiente, reasignando el resto entre otros regantes $\left(Q_{c}=\right.$ $Q_{v}+Q_{e}$ y $Q_{e}>0$ ). Cuanto mayor sea el valor máximo del agua ambiental, mayores serán las cantidades compradas y recuperadas para el medioambiente, y menor será la cantidad reasignada entre los agricultores (véanse las Figuras 3a-c). La agencia que administra el banco de agua ejerce su poder de mercado para crear un diferencial entre los precios de compra y venta $\left(P_{c}<P_{v}\right)$ como se muestra en las Figuras $4 \mathrm{~d}$-f $)$, lo que permite que el banco se autofinancie, puesto que no se necesitan fondos públicos para la adquisición de agua ambiental. En esta fase el valor del agua ambiental $V_{e}$ es mayor que el precio de venta $Q_{v}$, haciendo que mejore la eficiencia total. Durante esta fase, las ganancias de eficiencia resultantes de la mejora en los flujos en las masas de agua (medido como $\int_{V_{e}}^{V_{e}^{0}} Q_{e}\left(V_{e}\right) d V_{e}+Q_{e} V_{e}$ en la ecuación 4) superan la pérdida de eficiencia económica causada por la recaudación de fondos públicos a través de impuestos, cuantificados a través del coste marginal de los fondos públicos (Presupuesto $\times C M F)$. Por lo tanto, hay un aumento continuo en la eficiencia económica total $\left(E_{c}+E_{v}+E_{e}\right)$ como se muestra en las figuras $4 \mathrm{j}-\mathrm{I}$.

La tercera fase comienza cuando los valores de $V_{e}^{0}$ son lo suficientemente altos como para que el banco de agua necesite gastar fondos públicos para poder seguir aumentando la eficiencia económica mediante la recuperación de más agua para el medioambiente (véanse las figuras $4 \mathrm{a}-\mathrm{c}$ ). Es decir, durante esta tercera fase, el poder de monopolio-monopsonio no permite mejorar más la eficiencia total a través de recuperar más agua para el medioambiente, 
por lo que se necesita una cantidad cada vez mayor de fondos públicos para comprar más asignaciones para fines ambientales (ver figuras $4 \mathrm{~g}-\mathrm{i}$ ).

La sustitución gradual de $Q_{v}$ por $Q_{e}$ termina en un punto de $V_{e}^{0}$ donde el sector agrario no puede competir por la compra de asignaciones de agua con la demanda social de agua ambiental $\left(Q_{v}=0\right)$. Este punto define el comienzo de la cuarta fase, para valores de $V_{e}^{0}$ superiores a $0,35 € / \mathrm{m}^{3}, 0,53 € / \mathrm{m}^{3}$ y $0,75 € / \mathrm{m}^{3}$ para los distintos escenarios de disponibilidad de agua del $75 \%, 50 \%$ y $25 \%$, respectivamente (ver figuras $4 a-c$ ). Durante esta fase, todas las asignaciones de agua compradas a los agricultores se dedican al medioambiente $\left(Q_{v}=Q_{e}\right)$, respaldadas por una cantidad creciente de fondos públicos (ver figuras $4 \mathrm{~g}-\mathrm{i}$ ). Sin embargo, el poder de monopolio-monopsonio de la agencia del agua le permite mantener los precios de compra por debajo del valor ambiental marginal del agua $\left(P_{c}<V_{e}\right.$, como se muestra en las figuras $4 \mathrm{~d}$-f). Por lo tanto, observamos una tendencia creciente en la mejora de la eficiencia total (ver figuras $4 \mathrm{j}-1)$.

\section{Conclusiones y recomendaciones}

Podemos concluir que el banco de agua propuesto, para cualquier escenario de sequía y para cualquier valor social por el agua ambiental, resulta ser un instrumento útil para mejorar la eficiencia económica total derivada del uso del agua durante episodios de escasez coyuntural de agua. Se ha mostrado cómo el poder de monopolio-monopsonio del banco de agua propuesto es capaz de crear un diferencial de precios en el mercado de dotaciones para la agricultura que, para ciertos valores sociales por el agua ambiental, puede autofinanciar la adquisición de agua para el medioambiente.

Otra importante innovación de este estudio es la incorporación de las ineficiencias inherentes al uso del gasto público, medido a través del coste marginal de los fondos públicos (CMF), al realizar compras públicas de dotaciones (o derechos) de agua. Los resultados obtenidos sugieren que evaluaciones previas de la recuperación de agua pública para el medioambiente a través de instrumentos de mercado podrían haber sobreestimado las ganancias de eficiencia porque ignoraron las ineficiencias relacionadas con el gasto público. Se evidencia de esta manera que cualquier evaluación de políticas que implique la compra pública de asignaciones o derechos de agua debería tener en cuenta estas ineficiencias a través del coste marginal de los fondos públicos.

Finalmente, debe comentarse que los resultados de las simulaciones podrían estar sobrestimando los niveles de eficiencia alcanzados por el banco propuesto, dados los supuestos simplificadores considerados para la modelización del funcionamiento del banco. Por este motivo, sería conveniente que en futuros trabajos se perfeccionase la modelización realizada al objeto de considerar los costes de transacción inherentes en las operaciones de mercado, así como minimizar los sesgos de agregación construyendo modelos a nivel de subcuencas hidrológicas.

\section{Agradecimientos}

Este trabajo ha sido financiado por el Ministerio de Ciencia, Innovación y Universidades (MCIU), la Consejería de Economía y Conocimiento de la Junta de Andalucía y el Fondo Europeo de Desarrollo Regional (FEDER) a través de los proyectos de investigación IRRIDROUGHT (RTI2018-095407-B-I00) y FINAGUA (UCO-1264548). 


\section{Bibliografía}

Dahlby, B. (2008). The marginal cost of public funds: Theory and applications. Cambridge, MA, USA: MIT University Press.

Gutiérrez-Martín, C., Gómez-Limón, J.A. \& Montilla-López, N.M. (2020). Self-financed water bank for resource reallocation to the environment and within the agricultural sector. Ecological Economics, 169, 106493.

Hanak, E. \& Stryjewski, E. (2012). California's water market, by the numbers: Update 2012. San Francisco: Public Policy Institute of California.

Molle, F., Wester, P. \& Hirsch, P. (2010). River basin closure: Processes, implications and responses. Agricultural Water Management, 97(4), 569-577.

Montilla-López, N.M., Gómez-Limón, J.A. \& Gutiérrez-Martín, C. (2018). Sharing a river: Potential performance of a water bank for reallocating irrigation water. Agricultural Water Management, 200, 47-59.

Wheeler, S.A., Garrick, D., Loch, A. \& Bjornlund, H. (2013). Evaluating water market products to acquire water for the environment in Australia. Land Use Policy, 30(1), 427-436. 